\title{
Study of an Ultra-Compact, Repetitive Marx Generator for High-Power Microwave Applications
}

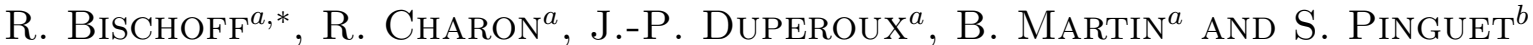

${ }^{a}$ French-German Research Institute of Saint-Louis (ISL), 5 rue du General Cassagnou, Saint-Louis, France

${ }^{b}$ Fachhochschule Gelsenkirchen, Neidenburgerstr. 10, Gelsenkirchen, Germany

The need for repetitive high-power microwave systems, for instance within the scope of convoy protection, requires the availability of compact, repetitive pulsed-power generators. The development and the first experimental results of an upgraded balanced ISL Marx generator for future repetitive operations at pulse repetition frequencies in the order of $100 \mathrm{~Hz}$ are introduced. A key objective is to keep the fundamental modular coaxial concept by reason of its scalability and compactness. Simulation models were developed under the PSpice software package in order to investigate the charging and discharging phases of the Marx generator and also to determine the design criteria for repetitive operations. The charging resistors were replaced by ultracompact inductors for rapid charging, being able to withstand pulsed voltages up to $60 \mathrm{kV}$ and pulsed currents up to $1.4 \mathrm{kA}$ during the discharge phase. An improved type of strontium-titanate high-voltage ceramic capacitor was successfully tested experimentally up to $70 \mathrm{kV}$. Each new elementary stage of the Marx generator consists of eight $1.1 \mathrm{nF}$ sectors of a cylinder capacitors mounted in parallel, two charging inductors of about $17.8 \mu \mathrm{H}$ and two halves of spherical spark gaps. The pressurized self-triggered gas switches are arranged along the axis for fast consecutive breakdown thanks to the UV radiation emitted during the breakdown in each gap. The stages are installed in individual fibreglass housings. First experimental results of a 4-stage Marx generator in repetitive operation, driven by a $4 \mathrm{~kW}$ capacitor charging power supply up to a maximum voltage of $40 \mathrm{kV}$, are presented.
\end{abstract}

PACS numbers: 84.30.Ng, 84.32.Hh, 84.32.Tt, 84.60.Ve

\section{Simulation}

The ISL ultracompact Marx generator using charging resistors, an in-depth description can be found here [1], represents the starting point of the development of the repetitive Marx generator.

The modeling of the charging phase of a resistive Marx generator shows that the charging time can be described by $t_{\mathrm{ch}}(N, R C)=a N^{2}+b N+c+d R C+e$, the formula's coefficients being given by the particular characteristics of the applied power supplies. This slightly differs from the empirical relation [2], although the results are comparable. The calculations show that the resistance values necessary for repetitive operations are unrealistic, for example about $225 \Omega$ for a pulse repetition frequency (PRF) of $100 \mathrm{~Hz}$, which is too low to prevent the discharging of the capacitor bank and consequently causes a significant loss of energy during the discharge mode. Previous experiments with the ISL Marx generator already showed massive problems at values of about $1 \mathrm{k} \Omega$. The last stages did not trigger reliably. This is also due to another problem related to resistive charging, i.e. the single stages are charged asynchronously. Therefore, replacing the resistive elements of the Marx ladder network by charging inductors is imperative for repetitive operation at pulse repetition frequencies of about $100 \mathrm{~Hz}$.

* corresponding author; e-mail: rainer.bischoff@isl.eu
Figure 1a shows the PSpice simulation scheme for the discharge phase of a balanced 7-stage Marx generator, which also considers the stray capacitors. The results
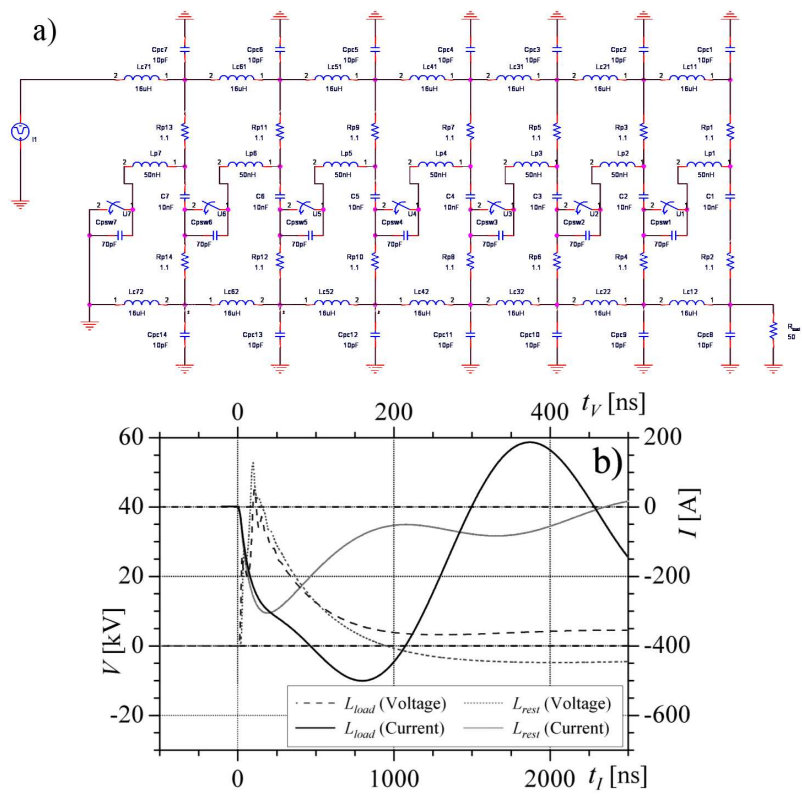

Fig. 1. (a) PSpice model of a balanced 7-stage Marx generator. (b) Voltage and current stress of the inductances during discharge. Higher stress on the inductance next to the load. $L=10 \mu \mathrm{H}, V_{\mathrm{ch}}=50 \mathrm{kV}$. 
of the influence of the charging inductance on the temporal profile of the output voltage make it clear that a charging inductance with a minimum value of about $10 \mu \mathrm{H}$ is necessary and are in good agreement with other simulations [3]. Figure 1b presents the temporal voltage and current stress of the charging inductances during discharge. It is obvious that all inductances must be able to withstand the full charging voltage, while the last charging inductance next to the load $L_{\text {load }}$ is exposed to a significantly higher current stress than the other inductances $L_{\text {rest }}$.

\section{Marx generator design}

The hand-made ultracompact charging inductors have a unit value of $17.8 \mu \mathrm{H}$, consisting of 3 layers, each of them with 16 windings, in cylindrical geometry of a HV-cable with a diameter of $1.0 \mathrm{~mm}$. This assembled coil measures only $20.0 \mathrm{~mm}$ in height and $18.2 \mathrm{~mm}$ in diameter. Despite the use of a cable with a DC-rating of only $18 \mathrm{kV}$ and $0.5 \mathrm{~A}$, the inductances are able to withstand pulsed voltages up to $60 \mathrm{kV}$ and pulsed currents up to $1.4 \mathrm{kA}$ for a pulse width of about $1.2 \mu \mathrm{s}$.

The capacitors by AVX consist of an optimized composition of strontium-titanate dielectric and have a special sector of cylinder geometry. They have been successfully tested up to $70 \mathrm{kV}$, exhibiting a loss in capacity $\Delta C / C$ of only $9 \%$ at $50 \mathrm{kV}$ and $15 \%$ at $70 \mathrm{kV}$.

Figure 2a shows an elementary stage of the new repetitive Marx generator. Each stage consists of eight 1100 pF capacitors in parallel, two charging inductors and two halves of the spark gaps. The electrodes are made of stainless steel spheres of $12 \mathrm{~mm}$ diameter. The electrode gap spacing is about $4 \mathrm{~mm}$. The parts are connected to custom-made horseshoe-type contact plates. Unlike earlier ISL Marx stages [1], the components are no longer irreversibly embedded in an electrically insulating epoxy resin but inserted in a special fibreglass housing to ensure the necessary electrical strength between the stages and from the stages to the ground. The fibreglass housing has a diameter of $200.0 \mathrm{~mm}$ and a height of $36.0 \mathrm{~mm}$. The elementary stages are electrically connected with multi-contact pin elements. The fibreglass housing is O-ring-sealed, which allows the volumes of the spark gaps on the axis and those of the $\mathrm{HV}$-components to be operated with different gas types and gas pressures. A trigger pulse generator is available for the triggering of the first spark gap, although at the moment only for non-repetitive operation. Otherwise, the spark gaps are self-triggered. The fast consecutive breakdown inside the Marx generator is supported by the UV flashlight emitted during the breakdown in each gap, on account of the on-axis arrangement of the spark gaps.

The photograph in Fig. 2b illustrates the assembled 4-stage inductive Marx generator used for first experiments. The output voltage $V_{\text {out }}$ is measured with a commercial resistive thiosulphate voltage divider type Europulse EP3205 placed in a conical housing, which is
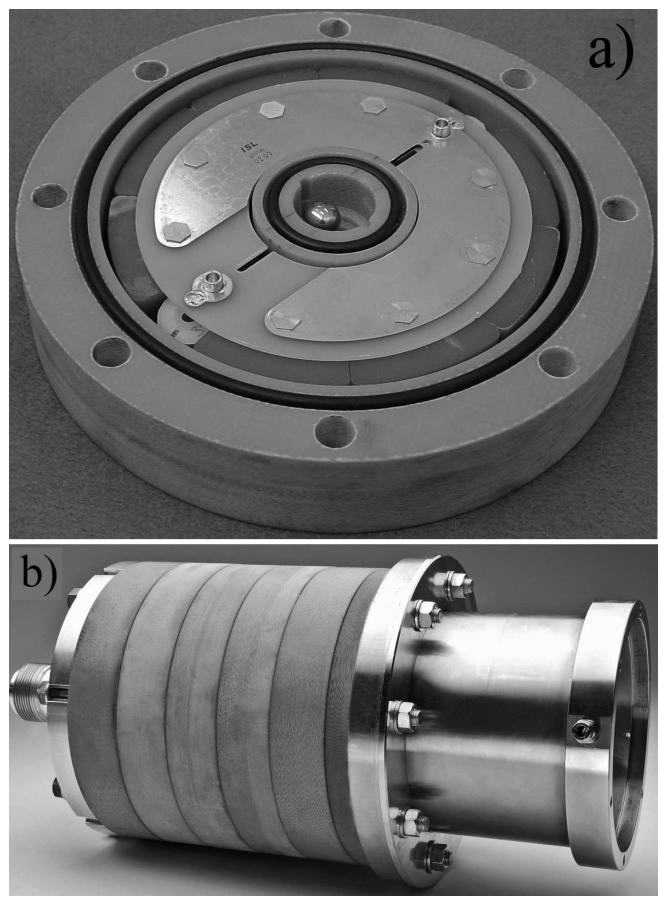

Fig. 2. (a) Complete elementary stage of the ultracompact repetitive Marx generator. (b) Assembled prototype of the 4-stage repetitive Marx generator with a coaxial output adapter.

mounted on the coaxial output adapter of the Marx generator. The voltage divider also serves as a load with a $50 \Omega$ impedance.

\section{Experimental results}

For the first functional tests the 4-stage Marx generator was connected to a $0-100 \mathrm{kV}, 300 \mathrm{~kJ} / \mathrm{s}$ power supply of the type Spellman SL300. Operating in single-shot mode, the Marx generator worked perfectly up to a charging voltage of $V_{\mathrm{ch}}=50 \mathrm{kV}$, as presented in Fig. 3a. The achieved peak output voltage was $V_{\text {out }}=165 \mathrm{kV}$, the efficiency $\eta=82.5 \%$. The rise time was $t_{\mathrm{r}}=14 \mathrm{~ns}$. The variation of the charging voltage did not affect the temporal profile of the output signal or the efficiency of the Marx generator either. The working pressure of the spark gaps ranged from atmospheric pressure at $V_{\text {ch }}=20 \mathrm{kV}$ up to $0.38 \mathrm{MPa}$ at $V_{\mathrm{ch}}=50 \mathrm{kV}$. The operating gas was technical air. The high-voltage parts were pressurized with $\mathrm{SF}_{6}$ at $0.30 \mathrm{MPa}$.

For repetitive charging the Marx generator was connected to a $0-40 \mathrm{kV}, 4 \mathrm{~kJ} / \mathrm{s}$ ALE power supply. The power supply worked in inhibit-mode, which allowed the external adjustment of charging time, charging frequency and number of pulses during the burst mode. A burst sequence of two pulses was used to investigate the inter-pulse recovery behavior of the spark gap arrangement inside the Marx generator. Figure $3 \mathrm{~b}$ displays the output voltages for an inter-pulse delay time of $\Delta t=10 \mathrm{~ms}$, 

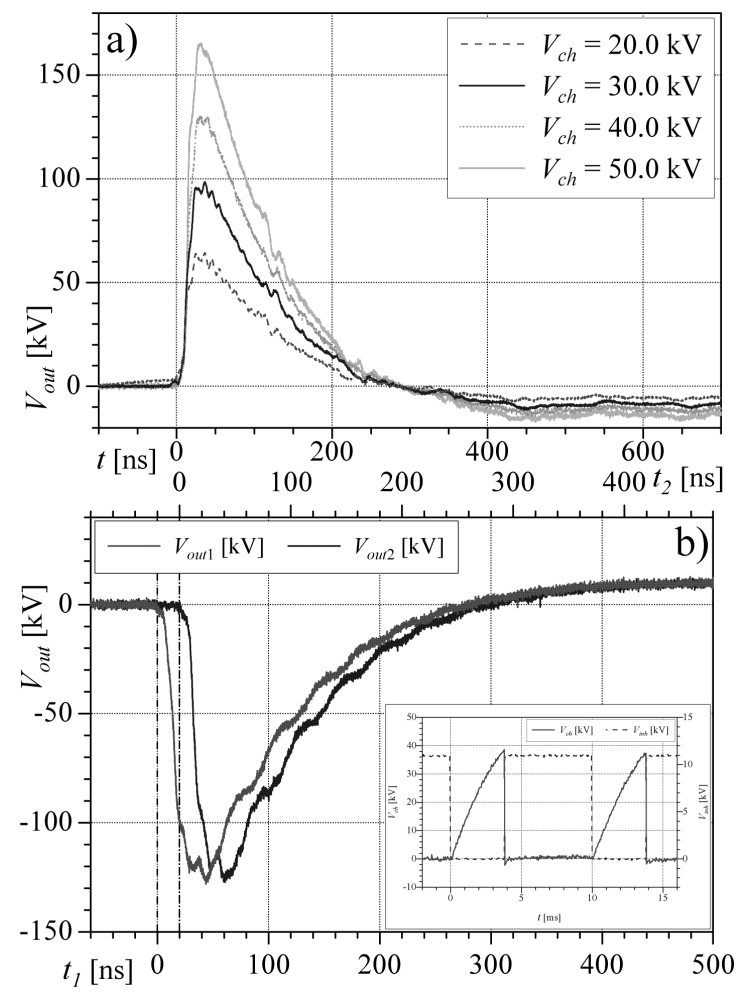

Fig. 3. (a) Output voltage $V_{\text {out }}(t)$ of the 4-stage Marx generator as a function of the charging voltage $V_{\mathrm{ch}}$. (b) Spark gap recovery test using a two-pulse burst. Full voltage recovery at a delay time $\Delta t=10 \mathrm{~ms}$, corresponding to $f=100 \mathrm{~Hz}$. The inset graph shows the charging and inhibit voltage. $V_{\mathrm{ch}}=38 \mathrm{kV}$.

which is the equivalent of a frequency of $100 \mathrm{~Hz}$. The spark gaps always exhibited a full voltage recovery at a working pressure of $0.32 \mathrm{MPa}$.

\section{Conclusions}

A modified design for the stages of an ultracompact repetitive Marx generator has been presented, adding new features such as easy maintenance and flexibility to its already existing high-energy capacity storage, compact coaxial structure, modularity and scalability. New charging inductors with an inductance $L=17.8 \mu \mathrm{H}$ and optimized HV ceramic capacitors in a sector of cylinder geometry have been introduced. The complete Marx generator has successfully been tested at charging voltages up to $V_{\text {ch }}=50 \mathrm{kV}$ in single-shot mode. Its efficiency amounted to $\eta=82.5 \%$ for a $50 \Omega$ load. First spark gap recovery tests indicate its ability to operate at frequencies of about $100 \mathrm{~Hz}$ and above.

Future investigations will include the implementation of a gas circulation and copper-tungsten electrodes to cope with heating and electrode erosion if operating the Marx generator in repetitive mode with a burst time of several seconds.

\section{Acknowledgments}

This work was funded by the "Bundesamt für Wehrtechnik and Beschaffung" (BWB) of Germany.

\section{References}

[1] B. Martin, P. Raymond, Rev. Sci. Instrum. 77, 043505 (2006).

[2] J.O. Loughlin, J. Lehr, D. Loree, in: Proc. 13th Int. Pulsed Power Conf., Eds. B. Reinovsky, M. Newton, 2001, p. 242.

[3] L. Veron, J.-C. Brion, in: Proc. 14th Int. Pulsed Power Conf., Eds. M. Giesselmann, A. Neuber, 2003, p. 1054. 